Document downloaded from:

http://hdl.handle.net/10251/81523

This paper must be cited as:

Diego-Mas, JA.; Alcaide Marzal, J. (2016). Single users' affective responses models for product form design. International Journal of Industrial Ergonomics. 53:102-114. doi:10.1016/j.ergon.2015.11.005.

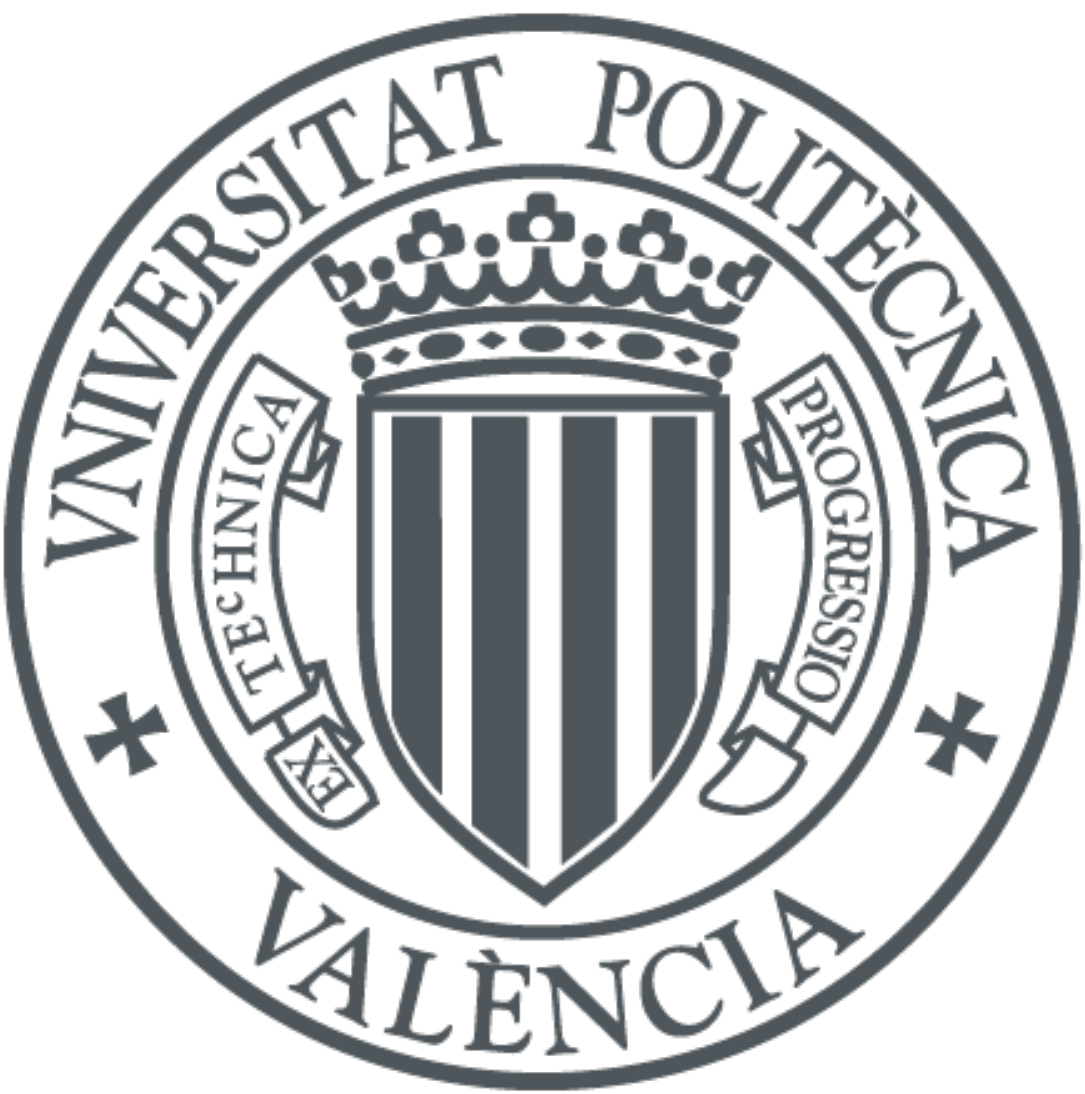

The final publication is available at

http://dx.doi.org/10.1016/j.ergon.2015.11.005

Copyright Elsevier

Additional Information 


\title{
Single users' affective responses models for product form design.
}

Jose A. Diego-Mas*, Jorge Alcaide-Marzal

I3B (Institute for Research and Innovation in Bioengineering), Universidad Politécnica de Valencia, Camino de Vera s/n, 46022 Valencia, Spain

Corresponding author:

E-mail address: jodiemas@dpi.upv.es (J.A. Diego-Mas)

Author version of the paper: Diego-Mas, J. A., \& Alcaide-Marzal, J. (2016). Single users' affective responses models for product form design. International Journal of Industrial Ergonomics, 53, 102114. https://doi.org/10.1016/j.ergon.2015.11.005

\begin{abstract}
This paper presents a neural network based approach to modeling consumers' affective responses for product form design. A theoretical framework for a single user's perception is developed. On the basis of this theoretical framework, a mathematical model which enables single users' responses to different products to be predicted was developed. The results obtained show that the mathematical models developed achieved highly accurate predictions.

For the purpose of obtaining a global model various individual mathematical models were created, which were based on the opinions of users representing different groups of opinion. The results suggest that, under some conditions, the combined use of various models of individual users can perform as well as a single model generated on the basis of mean market responses.
\end{abstract}

Keywords: user perception; product design; computational model

\section{Highlights}

- An approach to modeling consumers' affective responses for product form design is presented

- Several individual models are created for individual representative users

- Individual models are able to achieve great accuracy for individual users

- Several single models are used to generate a global model

- The approach presented seems to outperform a model created using mean responses 


\section{1 - Introduction}

The importance of the ability of a product to evoke emotions in the observer is becoming increasingly more important, since it has a decisive influence on purchasing decisions (Chuang and Ma, 2001; Desmet, 2003; Holbrook, 1985). In the current market a great variety of products of the same type can be acquired to sufficiently meet users' needs. Therefore, a product's shape, aesthetic features, visual appearance and ability to convey to the user the objectives for which it was designed, are all key to the success or failure of a product (Bloch, 1995; Chuang et al., 2001; Crilly et al., 2004). Additionally, sales platforms such as the Internet limit the user-product relationship to visual interaction alone, meaning that it is the appearance of a product which defines the image the user has of it (Dahan and Srinivasan, 2000; Vriens et al., 1998). This justifies the efforts carried out by many authors to provide models which match the attributes of a product to the consumers' affective responses (hereinafter CAR models). These models can be used to estimate how a user will assess a product in the early stages of the design process. A product's design can then be adapted to evoke the desired emotional response prior to its launch.

Hasdoğan (1996) studied the different types of user models and their role in product design. In some cases, such as Kansei engineering (Nagamachi and Imada, 1995), authors have aimed to translate the users' feelings into design attributes (Chang, 2008; Huang et al., 2012; Nagamachi, 2002; Schütte, 2002; Wang, 2014). Generally, the relationships obtained using these techniques are difficult to interpret, and do not enable functional models to be obtained which manage to predict the users' responses to certain design attributes (Han et al., 2000). For this reason, other approaches have been taken based on establishing conceptual frameworks of the customer's perception process in order to subsequently obtain mathematical models based on these frameworks. In other words, theoretical frameworks are created which define the mental processes shaping the users' image of the product, how judgments are formed with respect to products, and which external factors are influential. Once this conceptual framework has been defined, a mathematical model is obtained to model these processes.

Han \& Hong, 2003 contends that the user's affective response is based on a cause-effect relationship with the attributes of the product. In other words, certain product attributes lead to a certain user response. This is a basic assumption for the development of a CAR model, given that the model can be created by systematically analyzing the relationship between the users' responses and products' attributes (Hsiao and Chen, 2006; Yang and Shieh, 2010). Nevertheless, establishing such relationships is not easy given that there are several fundamental problems which must first be solved. The first problem is that the mental process carried out by the user from the time he receives the information regarding the product until the time he makes a judgment on it, is in practice, unknown. It is akin to a black box relating to which only the inputs and outputs are known. Only assumptions can be made about what happens inside the box. Therefore, the conceptual framework on which the CAR model is based is only validated, a posteriori, if the resulting mathematical model functions accurately. The processes that take place until the users make a judgment and the factors that affect the decisions taken 
vary from one model to another (Crilly et al., 2004; Engel et al., 1995; Han and Hong, 2003; Han et al., 2001). In order to partially solve this problem, methods which try to determine the user response without verbal assessment have been proposed (Ho and Lu, 2014; Lu and Petiot, 2014)

The second problem relates to how to codify the inputs and outputs of the black box model, in other words, how to define the product so that it serves as a model input, and how to define and measure the user's response. The product design should be transformed into a set of meaningful and measurable specifications. Therefore, it is necessary to determine which and how many product attributes define it completely, which of the attributes is relevant for the purpose of evoking an emotional response from the user, and how to measure them. Additionally, the relationship between the products' attributes and the users' judgment is not of a linear nature where there are strong correlations between the attributes. Therefore, it is difficult to process such information in order to generate a model (Park and Han, 2004; Shimizu and Jindo, 1995; Yang and Shieh, 2010).

Another fundamental problem relates to determining the mathematical technique whose use is most appropriate for obtaining the model. This problem has been approached from different angles. Traditional market response models are based on statistical techniques such as the multiple linear regression technique, logistic decline, discriminant analysis, etc. Nevertheless, more recent approaches tend to rely on techniques which are more flexible such as the Artificial Neural Networks (ANNs), or semiparametric regression models, fundamentally due to their ability to find the non-lineal relationships which exist between variables. ANNs are used in numerous marketing and product design fields like market response prediction, segmentation or consumer choice prediction. Various studies have been carried out to compare the results obtained using statistical and ANN response models (Dasgupta et al., 1994; Paliwal and Kumar, 2009; Venugopal and Baets, 1994; West et al., 1997). In these studies, the capacity of models based on ANNs outperforms that of models based on statistical methods. This occurs, for example, in ten of the twelve comparative studies analyzed (Paliwal and Kumar, 2009). Consequently, most of the recent research in this field tends to rely on ANNs (Chen et al., 2002; Ishihara et al., 1997; Lai et al., 2006, 2005; M. Shieh et al., 2008; M. D. Shieh et al., 2008; Yang and Shieh, 2010)

However, the fundamental problem relating to the development of a CAR models stems from the variety of different users' opinions regarding a single product. Generally, conceptual frameworks are based on the premise that there is a cause and effect relationship between the attributes of the product and the user's response. Nevertheless, these relationships vary from one user to the next since their opinions are not based entirely on the attributes of the object. Individual and external conditioning factors such as personal taste, cultural environment, level of education, and personal motivations and aims will all lead the perception of each user to vary (Allenby and Ginter, 1995; Chai et al., 2015; Engel et al., 1995; Hoch et al., 1995). However, this does not mean that the existence of a cause and effect relationship between the product's features and the user's response should be ruled out. These conditioning environmental factors should simply be considered as characteristics of the user himself. 
To take the above into account, conceptual frameworks consider the user in addition to personal and environmental conditioning factors. However, the mathematical models developed based on these frameworks do not usually reflect this approach. For example, mathematical models tend to look for relationships between product attributes and the mean responses of a representative sample of users. It can be said that these models attempt to predict the opinion of an average user. Although it can be concluded that there is a causal relationship between the appearance of an object and the consumers' mean responses, these relationships are much more complex than the relationships for the opinion of a single user. In the sample of users taken into account for the development of the model, a user whose opinions regarding a product are similar in all cases to the mean market responses might be impossible to find. Average opinion consists of the result of the judgment of users whose ways of perceiving product attributes might be different and whose way of assessing them are definitely different. Consequently, these CAR models do not correspond to the conceptual framework on which they are based.

The development of these models requires a lot of effort and survey time. Many users and many responses by user are necessary to generate the data to obtain the statistical models. This could be a big problem because respondents' boredom and fatigue could lead to obtain unreliable data. This study shows a different approach to generate a CAR model that looks for reducing the amount of time and effort to obtain a market model and considers the previously exposed problems in the development. For this, a conceptual framework of the user perception process was first proposed. This theoretical framework took into consideration the personal and environmental factors conditioning the judgments made by each user. Therefore, in practice the CAR model obtained based on this framework would only be valid for one user. However, although the perceptual relationships to be modeled are different for different users, if the opinions of a group of users regarding a selected sample of products are similar enough, it can be concluded that their perception processes and specific conditioning factors are similar. Consequently, by grouping users based on the similarity of their judgments, a mathematical model can be generated for a user representative of each one of those groups. With a certain margin of error, the CAR model would be valid for all users included in this cluster. As opposed to the traditional approach, in which a model predicting the mean response of the users in the cluster would be created, a model based on the opinions of a user representative of the cluster was generated. A model created in this way takes into consideration the individual conditioning factor affecting the way in which the product is perceived. By generating a model for each group of users with similar opinions, the mean market response was able to be determined. For this purpose, the response from each model was weighted by the relative size of the cluster containing the user from which the model was obtained.

The question posed in this study was whether a model based on opinions of individual users who are representative of various groups of users could be exact enough to avoid the development of a more time consuming model based on the mean opinion of all users. Additionally, the model based on 
this new approach was tested to determine if it reached a high enough degree of generalization enabling it to be used to predict different judgment of users on different types of products.

For this purpose, the paper was structured as follows: the first section briefly describes the conceptual framework of the product perception process which was created in this study as a basis for the CA model. The second section briefly explains the way ANNs and Genetic Algorithms (GAS) operate, being that they were used to develop the mathematical model. The final section describes the mathematical model developed and how it was applied to two case studies, in addition to the results and conclusions made.

\section{2 - Proposed conceptual framework}

Industrial products possess a number of attributes which are perceptible to the senses of the user and can be characterized at different levels. The number of attributes and attribute levels which are relevant in shaping users' opinions cannot be determined a priori. Tools such as Conjoint Analysis (Green and Rao, 1971; Luce and Tukey, 1964) can aid in determining the importance of each attribute. The information relating to these attributes and attribute levels reach users through their senses. The user assesses the product based on these attributes. The mental process that takes place within the user is a "black box", but is possible to theorize about what occurs inside the box. The assumptions made are used to create the CAR model, and they are only considered to be valid if the model finally obtained based on these assumptions is capable of predicting the users' responses.

Three process stages can be identified: the first two give rise to the sensation provoked in the observer by the product, while the third leads to the perception and assessment of the product (Figure 1). It is necessary to differentiate between sensation and perception, two concepts that will be used later. Sensation can be defined as the impression that things give through the senses, immediately after the physiological excitement produced. It is a basic and immediate experience generated by simple remote stimuli (Foley and Matlin, 2010). Perception can be defined as a person's mental interpretation of sensations, which involves organizing them and giving them meaning and requires the active participation of the brain (Feldman, 2013). Evidently, perception follows sensation.

\section{Stage 1: Reception and filtering of information.}

The user receives information regarding the product through his senses. Although the information received is the same for all users, the information which is processed can be different for each user. This is due to the specific conditioning factors that exist: physiological (the capacity of the senses to perceive details or be trained to perceive certain attributes), psychological (the state of mind of the observer), physical (the environment and way in which the product is presented) and social (education, cultural level, etc.) Once received the information is filtered. Not all of the product attributes will have the same impact on the sensation evoked in the user. In this stage superfluous information is eliminated whereas the relevant information passes on to the next stage, and is weighted by the importance it has on the generation of the sensation provoked in the user by the product. 


\section{Stage 2: Pre-processing of information and the generation of sensations.}

The information received in stage 1 is processed to produce sensations. In this stage the information relating to the product attributes can be combined to give rise to new combined attributes, which can make a difference in the sensation produced. Therefore the information relating to the color of the product combined with information on the texture of the materials or its shape, can give rise to a more generic attribute which could be called "appearance". Consequently, it can be concluded that there is an integration of data which adds global information relating to the product to the information received through the senses. In this way the product generates a sensation in the user resulting from the preprocessing of the information reaching the user through his senses.

\section{Stage 3: Perception and rating}

Having produced the aforementioned sensation based on the pre-processed information, the perception process was initiated. Perception is seen as the process by which a global mental image of the product is obtained. To obtain this global mental image the user makes a number of parallel assessments regarding the sensation that the product produced. For example, he would rate the product as being useful: how much does it cover my needs?, or as being a valuable possession: how much do I want to own this product? or as having aesthetic value: To what extent do the levels of the different attributes adapt to my preferences?

Because users have obtained the mental image of the object through their perception, it is possible to request that they make a judgment regarding a certain aspect of the product. Assuming that the user is asked to rate how much he likes the product observed on a Likert scale, the user would use his global image of the product to assess the degree to which he likes the product, and finally he would provide a response.

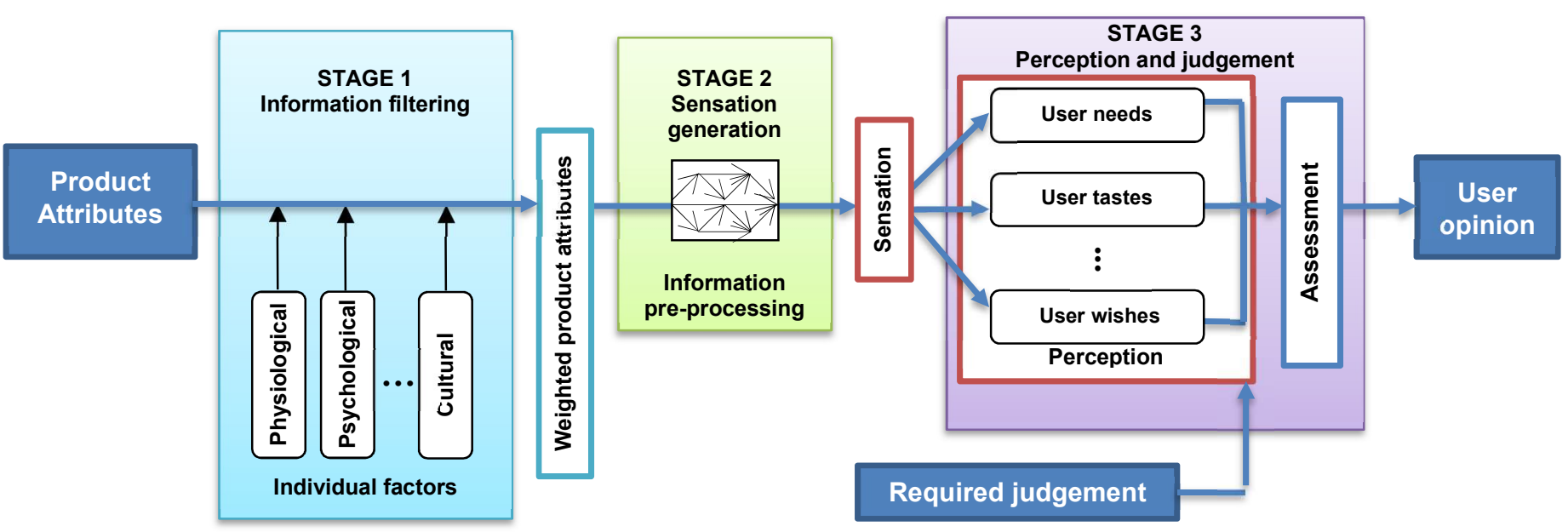

Figure 1. Proposed conceptual framework of the product perception process 
As previously discussed, the three stages of this process are conditioned by factors that can vary from one user to the next, the physiological and psychological characteristics of the individual, their educational level, their cultural origin, the way in which they integrate the information received through the senses, the weight given to their functionality, the aesthetics or the brand image of the product, etc. Therefore, it is necessary for the mathematical model developed from this conceptual framework to take into account these particularities. ANNs and GAs were used to develop the CAR model. Therefore the manner in which they operate is explained in the next section.

\section{3 -Neural networks and genetic algorithms}

An ANN is a mathematical model that represents a distributed adaptive system built by means of multiple interconnecting processing elements, just as real neural networks do. ANNs are used in many fields of research (psychology, robotics, biology, production or computer science, to name a few) (Principe et al., 2000) due to their ability to adapt, learn ${ }^{1}$, generalize, organize or cluster data. Given their ability to learn (in comparison with sequential systems), they are instruments which are suitable for generating models such as those described in the previous section, there being various uses of ANNs in this environment (Chen and Yan, 2008; Dasgupta et al., 1994; Hsiao and Tsai, 2005; Ishihara et al., 1997; Lai et al., 2006, 2005; Tsai et al., 2006; Yang and Shieh, 2010)

The processing elements (neurons) are distributed in several layers (Figure 2). The intermediate layers are known as the hidden layers, while the first and the last layers are known as the input and output layers, respectively. In general terms, each neuron receives signals processed and transmitted by neurons in the preceding layer and in turn processes and transmits them on to the next layer. The number of layers and the way in which the neurons are connected determine the architecture of the network.

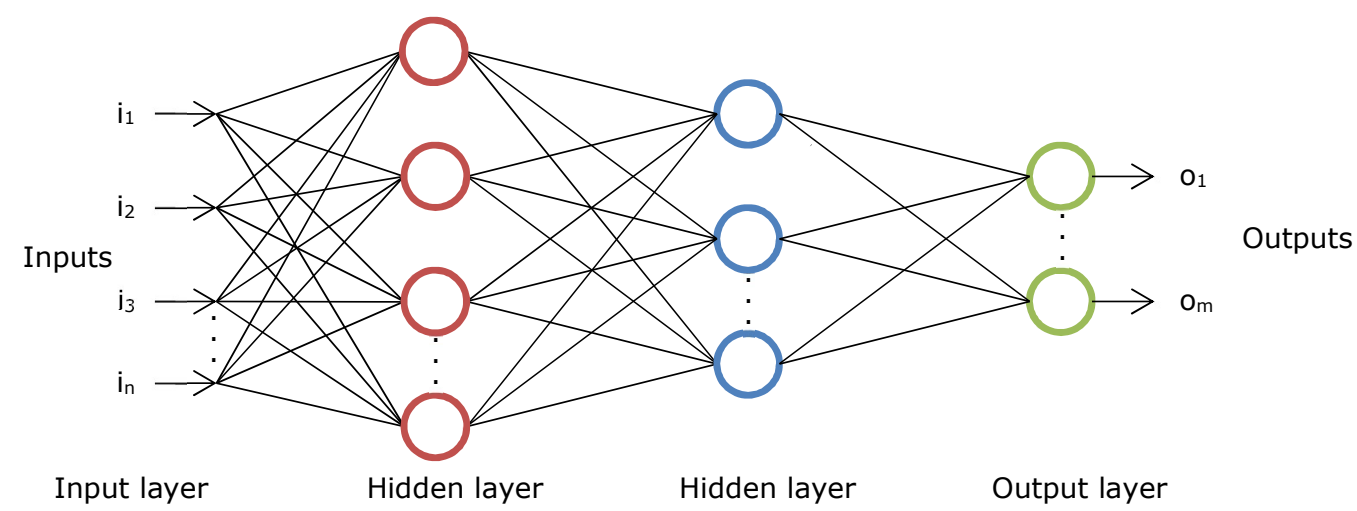

Figure 2: Example of the structure of a neural network with two hidden layers, $n$ inputs and $m$ outputs.

\footnotetext{
${ }^{1}$ Neural network learning or training is an adaptive procedure in which the weights of the connections between neurons are incrementally modified so as to improve the network performance until reaching a specified criterion.
} 
The input signals $\left(i_{1}, i_{2}, \ldots, i_{n}\right)$ are the values of the variables representing an instance of the phenomenon to be modeled (e.g. product attributes). They are collected by the input layer which transmits them through links to the neurons in the first hidden layer. The signals are scaled in each link according to an adjustable parameter associated with each connection between neurons called weight. Usually, the initial weight of each link is randomly set. Each neuron in the hidden layer collects the signals from the connections, adds them up and produces an output that is a function of the sum. The most commonly used functions are sigmoids, hyperbolic tangents and linear versions of the latter. The signals traverse the network from the input layer to the output layer, where the network response to the inputs is collected $\left(\mathrm{o}_{1}, \mathrm{o}_{2}, \ldots, \mathrm{o}_{\mathrm{m}}\right)$.

Supervised learning networks are able to learn the relationships between the inputs and outputs through the repeated presentation of input data and the values of the corresponding outputs. Once trained, the network can generalize these relationships to new cases. The training process consists of presenting the network with a sufficient number of input cases and the desired output values. The output obtained by the network in each case is compared against the desired output, and the network error is calculated. Then, the weights of neuron connections are modified according to the selected training algorithm in order to minimize this error. This process is repeated until a criterion previously established is reached, for example, when the error value gets to a threshold or stops decreasing. Although there are different training algorithms applicable to different types of networks, the most commonly used to train ANNs is Back-Propagation (BP) (Rumelhart et al., 1986).

A significant problem relating to the development of ANNs is overfitting. Overfitting (also known as overtraining) occurs when a model captures the statistical noise in the data rather than the underlying signal (Sarle, 1995), i.e. the model memorizes the correct responses to each pattern rather than learning the relationships between inputs and outputs. When this happens, the model produces very accurate results with the data employed to train the network, but it is not able to generalize them to new cases. To avoid overfitting some regularization procedures can be used such as jitter, weight decay or early stopping. Early stopping is the use of a reduced data set (validation set) to calculate model error periodically during training. These validation data sets are not used to train the network, but rather to determine the moment when the model stops learning and starts memorizing the relationships between training patterns and their resulting outputs. The usual procedure is to divide the available data into three sets: the training set, which is used to train the network; the validation set, which is used to determine the early stopping point; and the test set, which is used to validate the degree of generalization of the trained model.

The architecture of the ANNs must be determined. The size of the network (number of hidden layers and neurons per layer) affects the model's capacity to generalize. Establishing the appropriate number of neurons is a major problem for which there is no systematic procedure, although there are some simple rules, such as the Masters rule (Sarle, 1995). In the hidden layers, the appropriate number of neurons will depend on the number of independent and dependent variables in the problem, the 
quantity and quality of the training data available, the complexity of the relationships between the input and output patterns, the type of neuron activation function and the training algorithm used.

The input variables of the model that have proved to be relevant, the number of networks layers, the number of neurons per layer and the activation function of each neuron, among other parameters, could be determined by means of GAs (Dam and Saraf, 2006; Kim et al., 2005). GAs perform a stochastic guided search based on the evolution of a set of structures as it occurs in natural species evolution (Goldberg, 1989). The starting point is a set of problem solutions called individuals. This first set is randomly generated and called initial population. Each individual is an ANN, and it is coded by a finite length chain called chromosome. Each individual solution is evaluated using an evaluation function to determine its suitability for the requirements of the problem. The population undergoes several transformations that yield a new population (new generation). These transformations are guided by some genetic operators, the most common being selection, crossover and mutation, which combine or modify the chromosomes representing the individuals. Crossover and mutation operators are applied to create a new generation of individuals that inherit the best characteristics of their predecessors. For this purpose, the individuals that will participate in each of the genetic operators, and those that will survive and pass on to the following generation, are selected previously by mean of the selection operator. The process is repeated with the new set of individuals until a certain number of iterations is reached, or until a certain number of iterations without a new best solution have been performed, making the individuals evolve to better solutions to the problem.

In this study a GA is used to determine which product attributes contribute towards shaping the users' opinions. Each individual will represent, inter alia, a combination of weights of the product attributes. This combination will be assessed based on the ability of the models generated to predict the users' responses. Simultaneously, the GA is used to determine the most appropriate topology of ANNs used in the model. Each individual will represent the parameters of an ANN (number of layers, number of neurons by layer, initial weights of the synaptic connections, step size, momentum, transfer functions, etc.). Each individual (that is to say, each ANN) will be assessed by the degree to which it is capable of predicting the opinions of the users once trained.

\section{4 - Mathematical approach to the model}

The perception process proposed in Section 2 was taken as a base, and a mathematical model was generated based on ANNs and GAs, to predict how users would rate a consumer product. As indicated there are certain conditioning factors that cause the sensation which is produced based on the information reaching us through our senses to differ from one user to the next. In order to solve this problem various individual models were generated which were valid only for a single user. Their use was subsequently generalized to obtain a global model representing their market opinion. Firstly we 
explain how to generate the individual models and the related training procedure required and, subsequently, how to obtain the global model.

\section{1 - Individual models}

The ANN based model which is presented will be valid for only one user. After training the ANNs with the data collected on a single user the neural network structure is capable of modeling the physiological, psychological, and physical particularities of the 'product, given that this information is implicit in the answers given by the user.

The model developed (Figure 3) is composed of two ANN, one unsupervised learning ANN and another parallel processing ANN. The information regarding product attributes, which has been appropriately filtered and weighted, reaches an ANN, which must then pre-process and combine it to give rise to new significant information (stage 2 of the perception process proposed in section 2), transforming the input samples into a new space (the feature space) where the information about the samples is retained, but the dimensionality is reduced. The type of network chosen was an unsupervised learning network which performs a principal component analysis (PCA). This network enables significant characteristics of a group of data which have not been previously classified to be differentiated since the network attempts to find redundancies and patterns internally based on which to group the information. The usual manner in which to carry out a PCA analysis is to analytically resolve an eigenvalue problem of the input correlation function. However, this analysis can be carried out by means of a single layer ANN trained with a Hebbian rule of learning (Oja, 1992, 1982; Sanger, 1989). An ANN of this type will have as many inputs as attributes established to define the product. The number of outputs will be determined during the training of the model. The outputs of the network are related to the eigenvalues and can be used as input to another neural network for classification. The appropriate number of outputs will be determined during the training of the model.

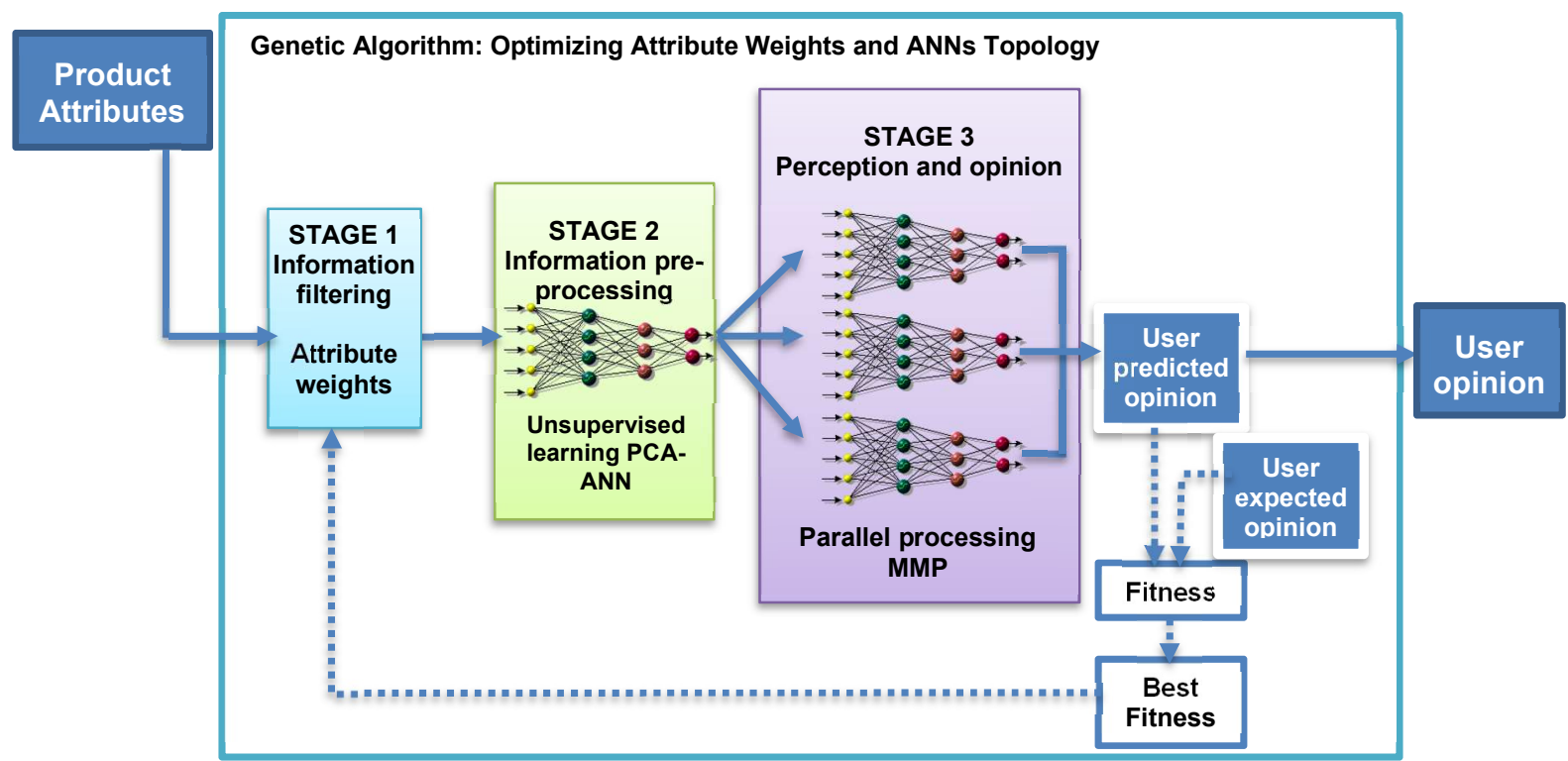

Figure 3: Mathematical model of the product perception and assessment process 
The outputs of the PCA-ANN will be used as inputs for a second ANN that will carry out the functions of stage 3 of the perception process proposed. The second ANN is a Modular Multilayer Perceptron (MMP). The networks are actually several networks which process the inputs in parallel and re-combine the outputs to obtain a common result. These independent networks which process the same input information tend to create certain specialized modules. Since the different networks are not connected together, except at the output, a smaller number of weights are required for the same size network. This tends to speed up training times and reduce the number of required training exemplars (Principe et al., 2000). This type of network was chosen because, as was indicated previously, to carry out the global assessment of the product, the user makes a number of parallel assessments which are subsequently combined into a single judgment.

\subsection{1 - Training of the model}

During the training of the model, it necessary to determine which ANN structure is most appropriate for solving the problems presented: to predict the user's response to a certain combination of product attribute layers. Specifically, the following should be established: the weight of each product attribute in the response, the number of PCA-ANN outlets (eigenvalues), the number of layers of the (MMP), the number of neurons by layer, the weights of the synaptic connections between the neurons and the type of transfer function of each neuron. Given that the number of parameters to be determined is large, it is advantageous to use solution-based metaheuristics to solve the problem. For this purpose, A GA is used during training to establish the most appropriate combination of parameters (broken lines of Figure 3).

The training process is as follows: after determining which product is to be analyzed, its most notable attributes and the level these attributes can take are established. Subsequently, a sufficient number of products of the same type are chosen and the level of each attribute is parameterized. The user who is to be modeled is interviewed after showing him each product and requesting that he make a judgment regarding the product The users' answers are grouped into three sets of data: the training set, which is used to train the model, the cross validation set, which is used during the training to avoid overfitting (see Section 3) and the test set, which is used to verify the adjustment of the model once trained Additionally, the test set is used to generate clusters of users in order to create the global model, which is described later.

The GA will create an initial population of random solutions for the problem of establishing the appropriate topology of ANNs. Each solution will be codified by means of a chain representing the weights of the attributes of the products and the different parameters of the two ANNs of the model (number of layers, number of neurons, transfer functions of each neuron, etc.). Each solution represents a different model that must be generated, trained and assessed, a process that is detailed later. When all individuals have been evaluated, a selection is made of those individuals in the population that will 
survive and pass on to the next generation. The new generation will be completed with individuals obtained through the crossover of the solutions of the previous generation. The best-fitted individuals of the existing population are used to breed a new generation using the crossover operator. For this process the roulette wheel selection method (Goldberg, 1989) is used. The mutation operator is applied to individuals selected at random among the individuals of the new generation. After having obtained the new population of solutions, the procedure is repeated until reaching a certain number of generations.

As already mentioned, the neural network of the model represented by each individual of the population should be trained to obtain its fitness. Therefore, for each individual of the population, a neural model is built with the topology codified in each solution and this model is trained. Since the model consists of two ANNs in series, one of which is of unsupervised learning, the training is carried out in two stages. During the first stage the information relating to the attributes of each product in the training set is entered. This information is used to train the PCA-ANN during a pre-set number of iterations. In a second stage, once the PCA-ANN has been trained, the weights of its connections will be set, and the transfer of the outputs of the PCA-ANN to the inputs of the MMP will be permitted, the result being the user's predicted response to the corresponding combination of product attributes. The degree to which the model is adapted will be determined by comparing the responses predicted by the model for each product to the responses given by a real user. The rule of learning used to train the MMP is Back-Propagation (BP) with momentum (Rumelhart et al., 1986). To prevent overfitting (see section 2) the cross validation set data are used to stop the process when reaching the early stopping point. After training has been completed, the test set data are introduced in the trained model, and the difference between the responses given by the model and those of the user are calculated. The degree to which the model is adjusted to the test set is then used by the GA as a basis on which to assess each of the population solutions. As previously stated, the test data are the responses that users have given to a certain number of products not used in the training phase. If the model is capable of offering a rating which is similar to the user's rating for these data, it will be considered to be a valid model. When the GA reaches a preset number of generations, the process is stopped and the best solution found is selected as a final model.

\section{2 - Global model}

The previously discussed model would enable us to predict the opinion of an individual user. The perception process varies from one user to another and a model generated from the opinions of one user would not be valid for another user. To obtain a tool enabling us to predict the opinion of whole group of potential users of the product, it is necessary to create an individual model for each different type of user comprised within the market. For this purpose, the whole group of potential users has to be clustered. 
In order to divide the users into different clusters the similarity of the judgments emitted with respect to the product chosen will be used. This is a critical issue in this approach. To obtain a good global model with this procedure it is necessary to be able to achieve well-defined user clusters. These clusters must be dissimilar between them and, at the same time, the opinions of the users inside each cluster must be similar. If this condition is not fulfilled, individual models will not be representative of the users in their cluster.

Once the users have been grouped according to the similarity of their opinions regarding the product, a representative user from each cluster can be selected. In this manner, an individual model can be obtained for each sector of market opinion. The output from each of these individual models will be similar to the opinion of the group which the user represents. The response of each individual model can be weighted by the size of its related cluster in order to obtain a global market model. Evidently, the size of the sample of users and the number of groups formed will have an influence on the exactness of the model when predicting market opinion.

Figure 4 shows the procedure used to obtain the global model. After having determined the type of product and the opinion for which a model is to be created, a sufficiently large and varied sample of products of this type is selected, and an image of each product is then obtained. Rather than using real products, images of the products can be used to develop the model. Using images of the products makes it easier to develop models without affecting the quality of the results, since photographic representation suffices to communicate most of the concepts in the same way that the real product would do (Artacho-Ramírez et al., 2008).

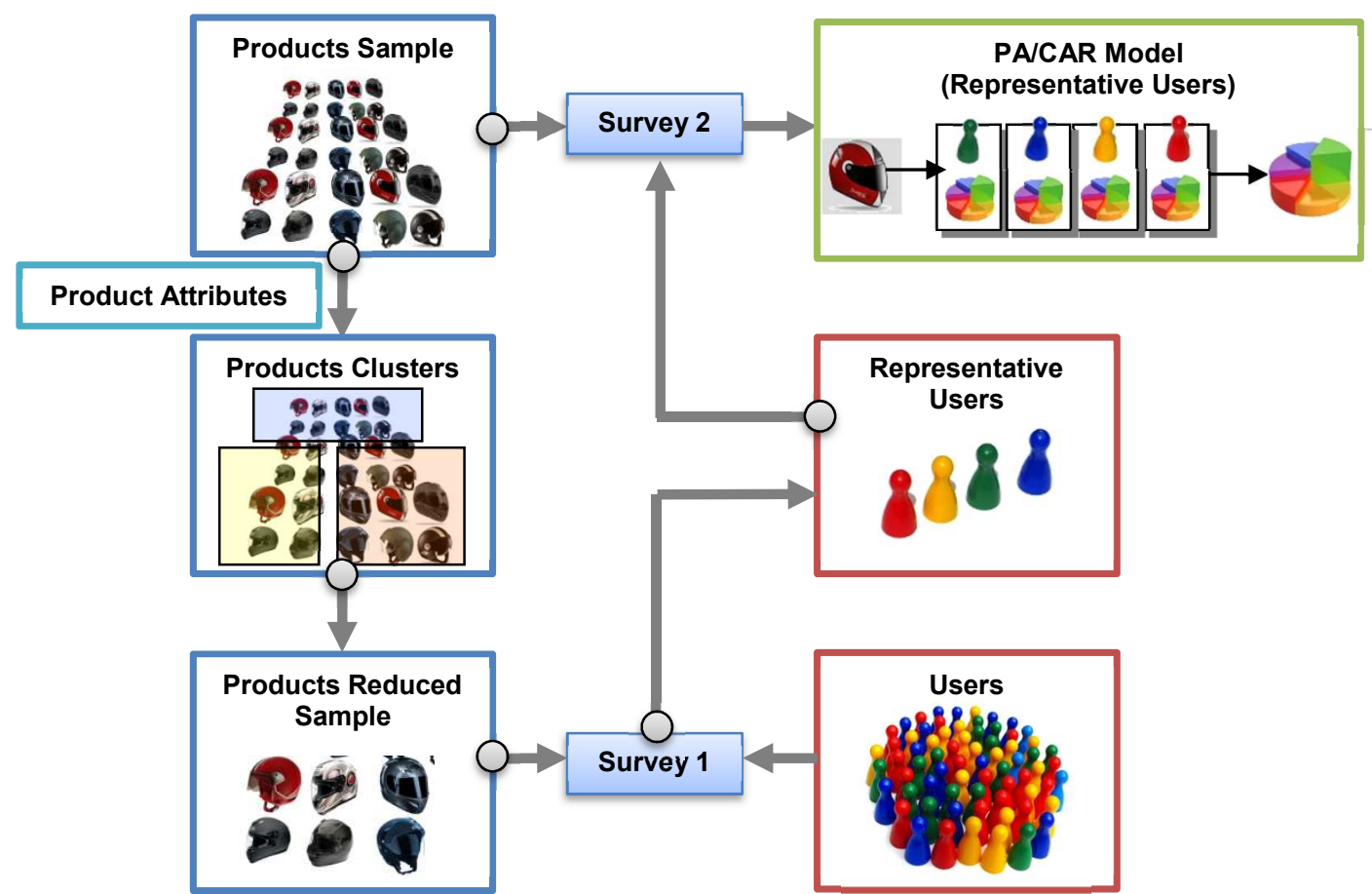

Figure 4: Procedure for the development of the global model.

After having obtained the images, the attributes defining the appearance of the product are determined. The attributes can be qualitative variables (such as colour) or quantitative (number of 
different colours). In the case of the qualitative attributes, the different levels for each of the attributes (red, green, blue, etc.) is then determined. The quantity of attributes should be sufficient to completely define each product. Each of the sample products is analyzed and the different levels for each of the attributes are then established, leading each product to be defined by the different levels for each attribute. After having determined the characteristics of the group of potential users for the product chosen, a sample which is both large enough and is representative of the users is selected. Although it is difficult to define the appropriate sample size for each study, according to (Mammasse and Schlich, 2014), Chambers \& Wolf (1996), sample sizes which are over 100 are generally considered appropriate for most market studies. In the experiments carried out in this study the sample sizes were always over 100 , both in the case of users and of products.

The criteria chosen to group the users is the judgment made with respect to the different products subject to study. To gather these opinions surveys are conducted in which the users are shown the images of the products and are then requested to make a judgment about them. Since the product sample size is large, it takes a long time for the users to respond to the survey. This can lead to fatigue, which reduces the reliability of the data obtained (Brace, 2013; Savage and Waldman, 2008). To prevent this problem the users who were interviewed are shown a smaller number of products representative of the different types available in the market. To choose the representative products, the products are grouped according to their attributes, and depending on the number of clusters, one or two products are chosen from each cluster. This grouping of products in clusters according to their characteristics enables a cross validation set and a test set for the training of individual models to be appropriately selected (see section 3). In these data sets, the highest possible number of product attribute levels should be represented so that an appropriate selection can be made by taking products from different clusters. The sample of representative products chosen for the purpose of surveying users was later used as a test set in the generation of individual models.

After having obtained responses from the users who were interviewed regarding the smaller sample of products, the potential product users can be grouped into clusters and a representative user from each group can be chosen. The results of this cluster analysis are used to test if the current approach is feasible. A long distance between the centers of the obtained clusters represents clear differences between the opinions of each users' groups. In addition, a short distance between the users and their cluster centers indicates that the users of each cluster employ a similar process to emit judgements, and then, using one representative user to obtain a single model of this cluster could be a good approach.

Each of the representative users is interviewed once again, and this time they are requested to give their opinion on the complete sample of products. To prevent the effects of boredom and fatigue, the surveys are carried out over different sessions and the survey takers are financially awarded. The data obtained is then used to obtain a model of each of the representative users, following the procedure described in the previous section. 
To obtain the model's global response to a product, the different attribute levels are introduced in the global model, and the number of different responses obtained is equal to the number of individual models generated. The response of each individual model will be considered to be the judgment of each cluster of users, thereby enabling an assessment of the product to be obtained which is segmented by user type. Given that the percentage of users belonging to each cluster is known, a single global response can be obtained. For this purpose, the mean responses of the individual models can be calculated weighted by the size of each cluster as compared to the total number of users.

\section{5.- Materials and methods}

Two case studies were used to determine the proposed model's ability to predict the users' specific judgments regarding the different consumer products and validate the assumption leading to the development of the model. The products were selected taking into consideration that a priori, the weight of the non-functional attributes had a significant impact on the users' assessments. The products chosen were paddle tennis rackets and motorcycle helmets. In the case of the paddle tennis rackets the question the users were asked was "Would you buy this racket?" This judgment was meant to determine whether the product's visible attributes trigger the user's impulse to purchase. In order to respond the user was required to assess the product globally. In the case of the motorcycle helmets, the users were asked "Does this helmet appear to be safe?" In this case the judgment to be made was more specific, requiring the user to assess one of the essential functionalities of the product (to offer protection to the user in the case of an accident) based on the visible attributes of the product. These two case studies were meant to validate the proposed method's ability to model the users' opinions regarding different products against general and specific judgments.

\section{1 - Case 1: Paddle tennis rackets}

Specialized publications and web pages were revised to obtain product samples which represent all the different types of the product and its most common attributes. Images of 130 paddle tennis rackets were gathered. Following their analysis and based on a study of the different types of rackets, 17 product attributes with a total of 48 possible attribute levels were identified. As an example, one of the attributes chosen was "racket shape". Based on the consultations of specialized bibliographical references, six possible attribute levels were assigned to this attribute (diamond, tear, round, mixed diamond/tear, mixed round/tear, undefined). An attribute level was then assigned to each of the 17 attributes for each of the 130 rackets.

The 130 rackets were clustered into groups based on their attribute levels in order to obtain a Reduced Product Sample in which all the different types of rackets were represented. For this purpose a TwoStep cluster algorithm (SPSS_Inc, 2007) was used. This procedure enabled clusters to be created based both on continuous and categorical variables and the automatic selection of the number of clusters. The TwoStep cluster algorithm was applied to the sample of 130 rackets, and the algorithm 
was enabled to determine the appropriate number of clusters, which was set at a maximum of 15 . The products were clustered via the Bayesian information criterion and the similarity among clusters was measured using multinomial probability distribution among the variables. As a result of this analysis, 13 clusters were established, each of which contained from 6 to 28 rackets. A racket from each cluster was chosen randomly to form the reduced product sample and test set that would be used to generate individual models. 30 rackets were selected to form the cross validation set (approximately $25 \%$ of the available data) so that all the clusters were represented in the data set. The remaining 87 rackets formed the training set.

\subsection{1 - Survey 1: Obtaining the reduced sample of users}

142 people were chosen to be interviewed ( 82 men and 60 women). All interviewees played amateur paddle tennis at least once every fifteen days and had been playing this sport for at least one year. Engelbrektsson (2002) and Karlsson, Kaulio, Hampf, \& Sperling (1998) concluded that experience and knowledge about a product were essential factors to assess specific matters about a product. Also, Schoormans, Ortt, \& de Bont (1995) suggest that product expertise allows customers to understand product information faster, to fill in missing information, and to discriminate the important aspects of the product. Thus, the sample was selected according to these considerations.

In order to conduct the survey, a web application was developed which enabled each product to be presented to the respondent in random order together with the judgment the respondent was required to make (Would you buy this racket?). The responses were to be given on a six-level Likert scale, and ranged from "Completely in agreement" to "Completely in disagreement". No neutral option was provided, and therefore respondents were forced to opt for one side of the scale. Subjects were permitted to take as long as they needed to answer the survey. The average amount of time it took to complete the rating of the 13 rackets forming part of the reduced sample of products was three minutes and 12 seconds. However, respondents were allowed to take a break if they considered it necessary. The subject's answers were numerically codified, being assigned a whole number ranging from -3 for "Completely in disagreement" to 3 for "Completely in agreement". The opinions of 142 potential users were obtained regarding 13 representative rackets representative of the different types of this product on the market. This information was used to group the users based on how similar their opinions regarding the products were.

For the purpose of obtaining the Representative Users, a k-means clustering analysis was carried out based on the responses given for each racket using SPSS, 16.0. This analysis was performed to obtain groups of users with homogeneous opinions. Cluster centers were automatically selected and updated after each assignment of a case to a cluster. The number of case reassignment reiterations was limited to 15 and the distance between cases was measured using a simple Euclidean metric. K-means clustering requires the specification of the number of clusters into which the cases are to be divided. Therefore, various analyses were carried out with different numbers of clusters. 
As aforementioned, the criteria for selecting the appropriate number of cluster was to obtain the maximum distance between the centers of the obtained clusters and a distance between the users and their cluster centers as shorter as possible. Considering the number of cases per cluster, the cases' distances from the cluster centers and the distances between the final clusters enabled six significant 6 clusters to be identified.

Convergence was achieved in the sixth iteration in which there was no change in the cluster centers. Two users were identified as outliers and were eliminated from the study. Given that the iterative resolution of the analysis was not invariable with regard to the order of the cases, the stability of each solution was evaluated by comparing the results of the same analysis with different orderings of the cases. The final clusters were formed by 34, 30, 26, 20, 17 and 13 users. To choose a representative user from each cluster, the distance of all the users from the clusters' centers was analyzed, the users closest to the center of each cluster being chosen. 6 Representative Users were obtained in this manner.

\subsection{2 - Generation of individual models.}

Each of the 6 Representative Users was interviewed and requested to take part in the study, for which they were financially rewarded. The surveys previously conducted were then repeated but on this occasion, the survey included a total sample of 130 rackets and it was conducted over a number of different sessions so as to avoid the effect of boredom. The rackets were presented to each user in random order in order to prevent the possible effect of having presented the products to the survey respondents in a certain order. The users' responses on a Likert Scale of -3 to +3 were standardized to range from -1 to +1 , and the responses were then used for the training of the individual models. Populations of 50 individuals were used in the GA, the objective function being to minimize the mean square error (MSE) of the cross validation data set. The MSE was measured on the outputs of the standardized model ranging from -1 to 1 . The crossover probability was set at 0.9 and the mutation probability at 0.01 . The maximum number of generations was 100 .

The GA was to determine the number of attributes to be used in training the model as well as the number of neurons in each layer of the ANNs. The number of attributes could range between a minimum of 5 and a maximum of 17. The GA was allowed to vary the number of neurons in each layer of the PCA-ANN (number of main components ) from 3 to 12. Sanger's learning rule (Sanger 1989) (also called Generalized Hebbian Learning) was used during unsupervised learning training stage. The MMP had two hidden layers and the number of neurons could range from 5 to 20 in the first layer and from 2 to 10 in the second layer. Additionally, the GA set the transfer functions for each neuron, which could be linear, hyperbolic tangent or logistic sigmoid. The MMP learning algorithm was Back Propagation with Momentum. The learning rate for the hidden layers could range between 0.1 and 0.4 , and between 0.1 and 0.2 for the output layer. Momentum ranged from 0.1 to 0.3 for the hidden layers and from 0.1 to 0,2 for the output layer. The maximum duration of the unsupervised learning phase 
was set at 5,000 epochs, with a learning rate of 0.01 decaying to 0.001 . The minimum network training passes for the MMP were 500, and the cut off was 5,000.

\subsection{3 - Results}

The average GA run time required to obtain each of the six individual models was three hours and 26 minutes on a PC with a $3.40 \mathrm{GHz}$ processor and 4 GB RAM. The characteristics of the set individual models found are shown in Table 1. The MSE of this table are the errors committed by the model when predicting the users' opinions on the test set products. These products were not used for training and therefore it was possible to measure whether the relationships between the product attributes and the users' opinions according to the models can be generalized to cases which were not used to obtain these relationships.

\begin{tabular}{|c|c|c|c|c|c|c|}
\hline & User 1 & User 2 & User 3 & User 4 & User 5 & User 6 \\
\hline Used Attributes & 13 & 16 & 10 & 14 & 15 & 11 \\
\hline PCA-ANN neurons & 8 & 11 & 7 & 8 & 9 & 6 \\
\hline MMP first hidden layer neurons & 13 & 15 & 14 & 16 & 15 & 13 \\
\hline MMP second hidden layer neurons & 7 & 5 & 7 & 8 & 6 & 7 \\
\hline \hline Test MSE & 0.4721 & 0.1722 & 0.1777 & 0.2639 & 0.3256 & 0.3855 \\
\hline
\end{tabular}

Table 1. Characteristics of the best models obtained for each Representative User.

The models obtained for each Representative User differ not only in terms of the weight of the connections among neurons, but also the number of main components of the PCA-ANN and the number of neurons in each hidden layer of the MMP. On the other hand, the activation function type of each neuron differs across models and across the neurons in a single model. It is normal to use the activation function of all neurons in the same layer. However, in this case, the GA was responsible for determining the most adequate type in each neuron. For example, the MMP of model 1 had 13 neurons on the first layer and 7 on the second. In the first hidden layer, the transfer functions were logistic sigmoids for 9 neurons and linear functions for the other 4 . In the second hidden layer, 5 neurons had hyperbolic tangents and 2 had a linear function. In the output layer the neuron had a linear function. Table 2 shows the opinions the users expressed on the 13 products of the test set (Desired) and those predicted by the corresponding model (Predicted). The test mean square error is shown in the last column.

\begin{tabular}{|c|c|c|c|c|c|c|c|c|c|c|c|c|c|c|c|}
\hline & Product & 1 & 2 & 3 & 4 & 5 & 6 & 7 & 8 & 9 & 10 & 11 & 12 & 13 & MSE \\
\hline \multirow{2}{*}{ User 1} & Desired & 1 & 1 & -2 & -2 & -1 & 1 & 1 & -3 & -2 & -3 & -1 & -2 & -2 & \multirow{2}{*}{0.4721} \\
\hline & Predicted & 0.6 & 0.84 & -2.41 & -1.87 & -1.54 & 1.12 & 0.35 & -1.84 & -1.23 & -2.54 & -0.05 & -0.82 & -1.23 & \\
\hline \multirow{2}{*}{ User 2} & Desired & -2 & -1 & 1 & -2 & 1 & -1 & -1 & -1 & -3 & -1 & -3 & -2 & 1 & \multirow{2}{*}{0.1722} \\
\hline & Predicted & -1.87 & -1.21 & 1.34 & -1.73 & 0.15 & -0.67 & -1.23 & -0.93 & -2.45 & -0.84 & -2.24 & -2.41 & 0.84 & \\
\hline User 3 & Desired & -2 & 1 & -3 & -2 & -1 & -1 & -2 & -2 & -1 & -2 & -2 & -3 & -2 & 0.1777 \\
\hline
\end{tabular}




\begin{tabular}{|c|c|c|c|c|c|c|c|c|c|c|c|c|c|c|c|}
\hline & Predicted & -1.42 & 0.87 & -2.56 & -2.21 & -1.42 & -1.21 & -2.04 & -1.27 & -1.14 & -2.34 & -1.58 & -2.39 & -1.47 & \\
\hline \multirow{2}{*}{ User 4} & Desired & -1 & -1 & -2 & -2 & 1 & 1 & -1 & -2 & -1 & 2 & -2 & -2 & -2 & \multirow{2}{*}{0.2639} \\
\hline & Predicted & -0.75 & -1.02 & -2.47 & -1.62 & 1.34 & 0.74 & -1.51 & -1.53 & -1.42 & 1.03 & -1.51 & -1.05 & -1.72 & \\
\hline \multirow{2}{*}{ User 5} & Desired & 1 & 2 & 1 & 1 & 1 & -2 & -2 & -2 & -2 & -2 & -1 & -1 & -3 & \multirow{2}{*}{0.3256} \\
\hline & Predicted & 1.35 & 0.84 & 0.92 & 0.74 & 0.61 & -1.27 & -2.21 & -2.32 & -2.42 & -1.53 & -1.47 & -2.07 & -2.69 & \\
\hline \multirow{2}{*}{ User 6} & Desired & -2 & 2 & -3 & -2 & -2 & 1 & -1 & -3 & 1 & -3 & -1 & -1 & -3 & \multirow{2}{*}{0.3855} \\
\hline & Predicted & -2.84 & 1.94 & -2.84 & -2.23 & -1.74 & 0.71 & -0.70 & -2.04 & 0.05 & -1.69 & -0.41 & -1.30 & -2.94 & \\
\hline
\end{tabular}

Table 2 Comparison of the uses' opinions on 13 paddle tennis rackets (Desired) and those predicted for the corresponding models (Predicted).

In order to predict the global rating it is necessary to calculate the mean of the values predicted for each individual model, weighted by the percentage of users represented by each model according to equation 1.

$$
P_{i}=\left(\sum_{m=1}^{N_{m}} p_{i}^{m} \cdot n_{m}\right) / N
$$

Eq. 1

Where:

- $\quad \mathrm{P}_{\mathrm{i}}$ is the global rating predicted for the product $i$.

- $\quad \mathrm{N}_{\mathrm{m}}$ is the number of individual models developed.

- $\quad \mathrm{p}_{\mathrm{i}}^{\mathrm{m}}$ is the assessment of product $i$ predicted for the individual model $m$.

- $\quad \mathrm{N}_{\mathrm{m}}$ is the number of users belonging to the cluster of users $m$.

- $\quad \mathrm{N}$ is the total number of users employed to develop the models.

In order to compare the values obtained in this way with those provided by a model trained with the mean responses of all users a new model was trained. On this occasion the procedure used in the previous section was repeated, but without first grouping the users in clusters. In this case the mean response of all the users for each product was used for training (figure 5).

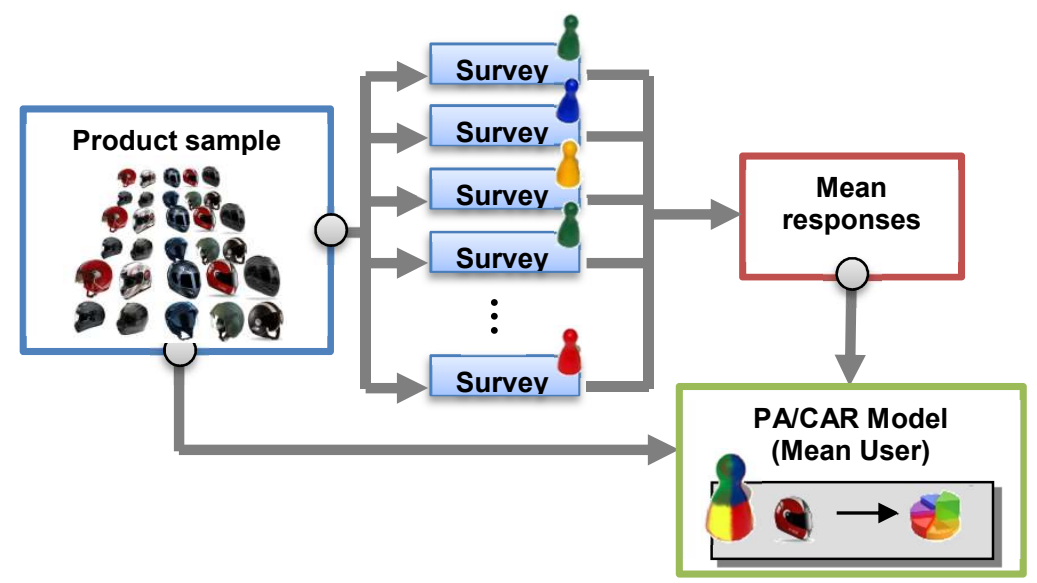

Figure 5 Procedure followed for the creation of a model based on the mean responses of the users 
Figure 6 shows the actual mean rating for each product in the Test Set (Desired), the average rating predicted by the model obtained through the approach taken in this study (Current Approach), and the rating predicted by the model trained with the mean responses of all the users (Mean Approach). The MSE of the model used in this study was 0.0591 , whereas when using the mean of all the users' responses it was 0.2157 .

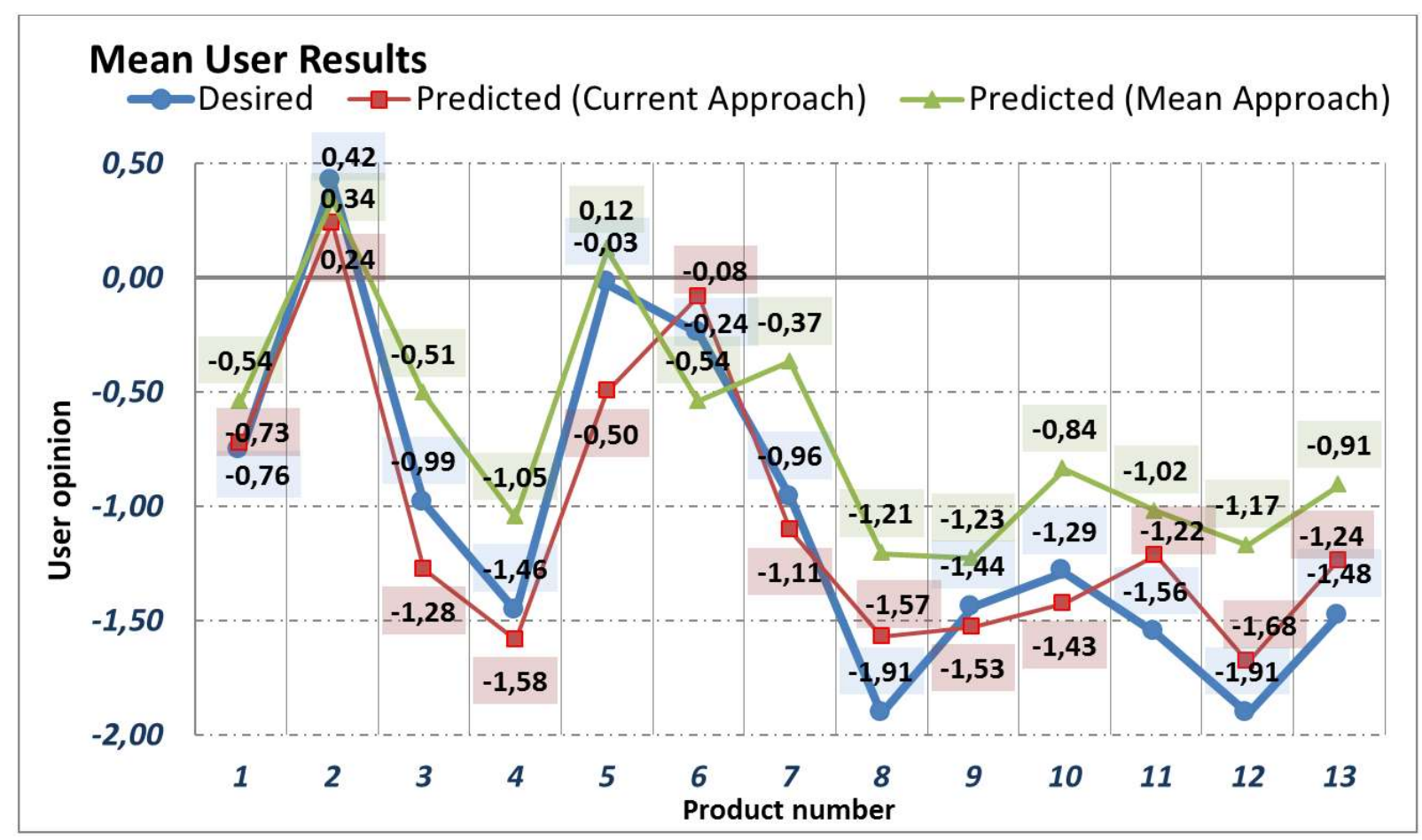

Figure 6 Rating of the 13 rackets by users (Desired) compared to the rating obtained with the mathematical models (Current Approach and Mean Approach).

\section{2 - Case 2: Motorcycle helmets}

The same procedure was followed as in the case of the paddle tennis rackets. Images of 133 motorcycle helmets were compiled, and a total of 29 product attributes were identified with 118 possible attribute levels. 133 helmets were grouped based on their attribute levels using the TwoStep cluster algorithm to obtain the Reduced Sample of the product. The analysis resulted in seven clusters with 26, 26, 21, $18,15,14$ and 13 helmets with similar features. Two helmets from each group were then randomly chosen as being representative of each cluster. Therefore, a set of 14 helmets was obtained with heterogeneous features representative of all the helmets. This set of helmets was also chosen as the test set for the training of the individual models. 33 helmets were selected to form the cross validation set (approximately $25 \%$ of the available data) so that all the clusters were represented in the data set. The remaining 86 helmets composed the training set. 
104 people were chosen to be interviewed ( 76 men and 28 women). Respondents were requested to say whether or not they agreed with the statement "The helmet you are seeing appears safe" for each of the 14 helmets in the Reduced Sample of the product, on a six-level scale. This information was used to build the users groups according to the same criteria as in the previous study case. Four clusters were obtained with 38, 36, 15 and 15 users, of which the four respondents closest to the cluster center were chosen as representative users. These users were shown a total of 133 helmets using the information obtained for the generation of 4 individual models.

\subsection{1 - Results}

The features of the best individual models found are shown in Table 3. The MSE of this table are the errors committed by the model when predicting the users' opinions on products in the test set.

\begin{tabular}{|c|c|c|c|c|}
\hline & User 1 & User 2 & User 3 & User 4 \\
\hline Used Attributes & 24 & 22 & 17 & 21 \\
\hline PCA-ANN neurons & 8 & 8 & 8 & 8 \\
\hline MMP first hidden layer neurons & 8 & 7 & 7 & 7 \\
\hline MMP second hidden layer neurons & 4 & 3 & 3 & 3 \\
\hline \hline Test MSE & 0.3549 & 0.4017 & 0.1618 & 0.6946 \\
\hline
\end{tabular}

Table 3. Characteristics of the best models obtained for each Representative User.

Table 4 shows the opinions the users expressed on the 13 products in the test set (Desired) and those predicted by the corresponding model (Predicted). The test mean square error is shown in the last column.

\begin{tabular}{|c|c|c|c|c|c|c|c|c|c|c|c|c|c|c|c|c|}
\hline & Product & 1 & 2 & 3 & 4 & 5 & 6 & 7 & 8 & 9 & 10 & 11 & 12 & 13 & 14 & MSE \\
\hline \multirow{2}{*}{ User 1} & Desired & 2 & -1 & 2 & -1 & 2 & 2 & -1 & -1 & -2 & 2 & 1 & 2 & 1 & -1 & \multirow{2}{*}{0.3549} \\
\hline & Predicted & 1.48 & -0.64 & 1.27 & -1.32 & 1.43 & 1.81 & -0.31 & -1.46 & -1.37 & 1.07 & 1.34 & 0.94 & 0.81 & -0.41 & \\
\hline \multirow{2}{*}{ User 2} & Desired & 1 & -1 & 1 & 3 & 2 & 2 & 1 & -1 & 3 & 2 & 3 & 2 & 2 & 1 & \multirow{2}{*}{0.4017} \\
\hline & Predicted & 0.74 & -0.3 & 1.98 & 2.04 & 2.57 & 2.54 & 0.21 & -0.76 & 2.48 & 2.59 & 2.27 & 2.34 & 1.3 & 0.64 & \\
\hline \multirow{2}{*}{ User 3} & Desired & -2 & -2 & 2 & 2 & 1 & 2 & -2 & -3 & 2 & 2 & 2 & 2 & -2 & -3 & \multirow{2}{*}{0.1618} \\
\hline & Predicted & -2.02 & -1.12 & 2.04 & 1.92 & 1.93 & 2.02 & -1.94 & -2.54 & 2.2 & 1.63 & 2.08 & 2.06 & -2.23 & -2.41 & \\
\hline \multirow{2}{*}{ User 4} & Desired & 1 & 1 & 2 & 2 & 3 & 1 & -1 & 2 & 2 & 2 & 3 & -1 & 1 & -2 & \multirow{2}{*}{0.6946} \\
\hline & Predicted & 0.31 & 0.85 & 0.94 & 0.80 & 1.85 & 1.25 & -0.76 & 1.78 & 1.45 & 1.26 & 1.61 & -0.05 & 0.14 & -1.77 & \\
\hline
\end{tabular}

Table 4 Comparison of the users' opinions on 14 motorcycle helmets (Desired) and those predicted for the corresponding models (Predicted).

As in the previous case study, a model was obtained by using the mean responses of all users for each product for training, without previously grouping the users into clusters. The global rating of each product predicted by this new model was compared to the rating obtained using equation 1 with the 
results of the individual models. Figure 7 shows the actual mean rating for each product in the Test Set (Desired), the average rating predicted by the model obtained through the approach taken in this study (Current Approach), and the rating predicted by the model trained with the mean responses of all the users (Mean Approach). The MSE of the model used in this study was 0.0772, whereas when using the mean of all the users' responses it was 0.1253 .

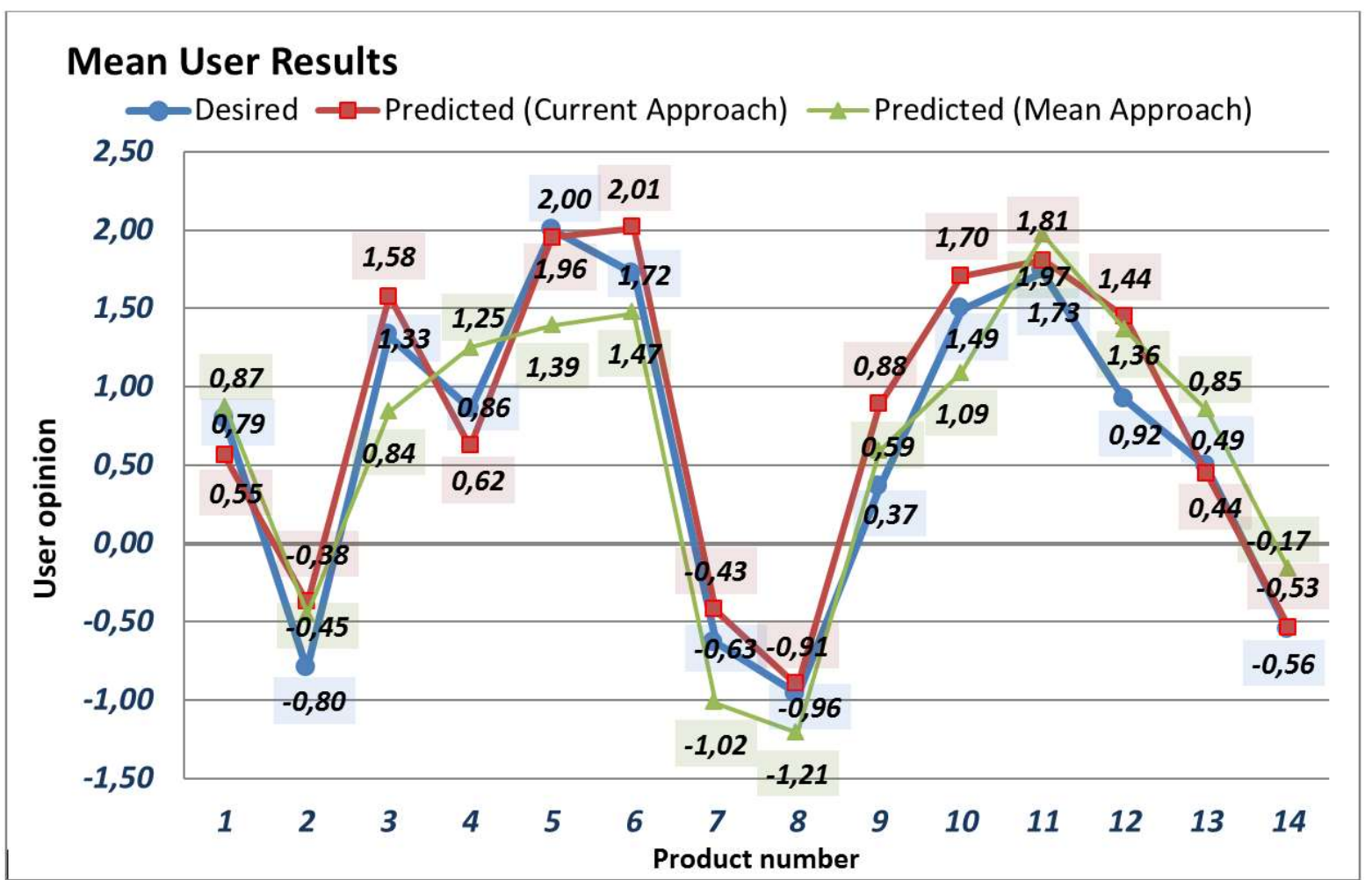

Figure 7 Rating of the 14 motorcycle helmets by users (Desired) compared to the rating obtained with the mathematical models (Current Approach and Mean Approach).

\section{6 - Discussion and conclusions}

The premise for this study was that there is a one-to-one relationship between a product's' attributes and a user's rating of it (Yang and Shieh, 2010). This relationship varies from one user to another, given that the users' own characteristics will have an influence on this relationship. Initially a conceptual framework of the perception process was proposed to model the response of a single user. This theoretical model was implemented in a mathematical model based on ANNs and GAs. In order to take the particularities of the various users into account in the mathematical model, different models were generated based on the responses of representative users of different groups of opinion.

In view of the results obtained by the mathematical model, it can be concluded that the conceptual framework on which it is based is valid. The individual models obtained in the two case studies are capable of predicting individual users' judgments based on the product attributes with enough accuracy. After having obtained the individual models, the mean response of the users can be obtained by weighting the responses of the individual models given the size of each cluster of users. In the case 
studies carried out, the models developed using this approach seem to be as accurate as those which attempt to directly model the mean response of all users. It should be recalled that the objective of this work was to develop a procedure to reduce the amount of time and effort to obtain a CAR model and not to obtain models outperforming other approaches. The fact that in the developed case studies the present approach slightly outperformed other approaches cannot be generalized, and it is not a conclusion of this study.

Usually, the aim pursued when generating a model is to determine the mean rating of a product, therefore, models based on the mean response of the users appear to be more focused from a mathematical point of view. This kind of models is specifically created to determine the relationship between a product's attributes and the mean responses of the users, meaning that this model will be highly adapted to the problem to be resolved. Nevertheless, this procedure does not take influential variables relating to the different users into account, and consequently the relationship between the product's attributes and the mean response of the users can be very complex and difficult to identify.

The approach taken in this study does not rely on the mean market responses but rather on individual opinions. Mathematically, this is a drawback because the model is not generated to obtain the mean market response, and this is accomplished in an indirect way. Moreover, the model does not use all the opinions of all the surveyed users; therefore, this approach offers less statistical power. However, the advantage of this approach is that the relationships between model inputs and outputs are simpler, since each individual model identifies relationships between the product attributes and the opinion of a single user. The results obtained show that this approach enables the mean response of users to be calculated with similar precision than mean approaches, the advantage being that the distribution of opinions in each cluster of users is known.

As stated before, several conditions must be fulfilled to develop models with this procedure. Welldefined user clusters are needed. These clusters must be dissimilar between them and, at the same time, the opinions of the users inside each cluster must be similar. If this condition is not achieved, individual models will not be representative of the users in their cluster.

The procedure described can be useful to product designers, since it enables them to know how a product will be perceived based on its attributes with less effort than other approaches. Additionally, it appears to adapt well to different types of products and to different ratings. A new design or a change in an existing design can be assessed quickly by the related model. However, there are some issues which require further research. A more comprehensive study on how the characteristics of the obtained clusters affect the accuracy of the model is needed. Moreover, it is necessary to consider whether certain types of products or judgments are more appropriate because they allow obtaining well-defined clusters. Additionally the clusters of users are formed based on opinions regarding the product analyzed. It would be advantageous to analyze if the users included in a cluster share other characteristics which are similar in addition to their opinion. 


\section{References}

Allenby, G.M., Ginter, J.L., 1995. Using Extremes to Design Products and Segment Markets. J. Mark. Res. 32, 392. doi: $10.2307 / 3152175$

Artacho-Ramírez, M.A., Diego-Mas, J.A., Alcaide-Marzal, J., 2008. Influence of the mode of graphical representation on the perception of product aesthetic and emotional features: An exploratory study. Int. J. Ind. Ergon. 942-952.

Bloch, P.H., 1995. Seeking the Ideal Form: Product Design and Consumer Response. J. Mark. 59, 16. doi: $10.2307 / 1252116$

Brace, I., 2013. Questionnaire design: how to plan, structure, and write survey material for effective market research, 3rd ed. London.

Chai, C., Bao, D., Sun, L., Cao, Y., 2015. The relative effects of different dimensions of traditional cultural elements on customer product satisfaction. Int. J. Ind. Ergon. 48, 77-88. doi:10.1016/j.ergon.2015.04.001

Chambers, E., Wolf, M.B., 1996. Sensory Testing Methods, 2d ed. ASTM Manual Series: MNL 26.

Chang, C.C., 2008. Factors influencing visual comfort appreciation of the product form of digital cameras. Int. J. Ind. Ergon. 38, 1007-1016. doi:10.1016/j.ergon.2008.04.002

Chen, C., Yan, W., 2008. An in-process customer utility prediction system for product conceptualisation. Expert Syst. Appl. 34, 2555-2567. doi:10.1016/j.eswa.2007.04.019

Chen, C.-H., Khoo, L.P., Yan, W., 2002. A strategy for acquiring customer requirement patterns using laddering technique and ART2 neural network. Adv. Eng. Informatics 16, 229-240. doi:10.1016/S14740346(03)00003-X

Chuang, M.C., Chang, C.C., Hsu, S.H., 2001. Perceptual factors underlying user preferences toward product form of mobile phones. Int. J. Ind. Ergon. doi:10.1016/S0169-8141(00)00054-8

Chuang, M.-C., Ma, Y.-C., 2001. Expressing the expected product images in product design of micro-electronic products. Int. J. Ind. Ergon. doi:10.1016/S0169-8141(00)00053-6

Crilly, N., Moultrie, J., Clarkson, P.J., 2004. Seeing things: consumer response to the visual domain in product design. Des. Stud. 25, 547-577. doi:10.1016/j.destud.2004.03.001

Dahan, E., Srinivasan, V., 2000. The Predictive Power of Internet-Based Product Concept Testing Using Visual Depiction and Animation. J. Prod. Innov. Manag. 17, 99-109. doi:10.1111/1540-5885.1720099

Dam, M., Saraf, D.N., 2006. Design of neural networks using genetic algorithm for on-line property estimation of crude fractionator products. Comput. Chem. Eng. 30, 722-729. doi:10.1016/j.compchemeng.2005.12.001

Dasgupta, C.G., Dispensa, G.S., Ghose, S., 1994. Comparing the predictive performance of a neural network model with some traditional market response models. Int. J. Forecast. doi:10.1016/0169-2070(94)90004-3

Desmet, P., 2003. A Multilayered Model of Product Emotions. Des. J. doi:10.2752/146069203789355480

Engel, J.F., Blackwell, R.D., Miniard, P.W., 1995. Consumer behavior, 8th ed. Dryden Press, Fort Worth.

Engelbrektsson, P., 2002. Effects of product experience and product representations in focus group interviews. J. Eng. Des. doi:10.1080/09544820110108917

Feldman, R.S., 2013. Understanding Psychology, 11th ed. McGraw-Hill, New York.

Foley, H.J., Matlin, M.W., 2010. Sensation and perception, 5ft ed. Allyn \& Bacon, Boston.

Goldberg, D.E., 1989. Genetic algorithms in search, optimization and machine learning. Addison-Wesley 
Longman Publishing Co., Inc, Boston, MA.

Green, P.E., Rao, V., 1971. Conjoint measurement for quantifying judgmental data. J. Mark. Res. 8, 355-363. doi: $10.2307 / 3149575$

Han, S.H., Hong, S.W., 2003. A systematic approach for coupling user satisfaction with product design. Ergonomics 15, 13-14.

Han, S.H., Hwan Yun, M., Kim, K.-J., Kwahk, J., 2000. Evaluation of product usability: development and validation of usability dimensions and design elements based on empirical models. Int. J. Ind. Ergon. doi:10.1016/S0169-8141(00)00019-6

Han, S.H., Yun, M.H., Kwahk, J., Hong, S.W., 2001. Usability of consumer electronic products. Int. J. Ind. Ergon. doi:10.1016/S0169-8141(01)00025-7

Hasdoğan, G., 1996. The role of user models in product design for assessment of user needs. Des. Stud. 17, 1933. doi:10.1016/0142-694X(95)00007-E

Ho, C.-H., Lu, Y.-N., 2014. Can pupil size be measured to assess design products? Int. J. Ind. Ergon. 44, 436441. doi:10.1016/j.ergon.2014.01.009

Hoch, S.J., Kim, B., Montgomery, A.L., Rossi, P.E., Montgomery, L., 1995. Determinants of Store-Level Price. J. Mark. Res. 32, 17-29. doi:10.2307/3152107

Holbrook, M.B., 1985. Emotion in the consumption experience: Toward a new model of the human consumer, in: Peterson, R.A., Hoyer, W.D., Wilson, W.R. (Eds.), The Role of Affect in Consumer Behaviour: Emerging Theories and Applications. Lexington, MA, pp. 17-52.

Hsiao, K. a., Chen, L.L., 2006. Fundamental dimensions of affective responses to product shapes. Int. J. Ind. Ergon. 36, 553-564. doi:10.1016/j.ergon.2005.11.009

Hsiao, S.-W., Tsai, H.-C., 2005. Applying a hybrid approach based on fuzzy neural network and genetic algorithm to product form design. Int. J. Ind. Ergon. 35, 411-428. doi:10.1016/j.ergon.2004.10.007

Huang, Y., Chen, C.H., Khoo, L.P., 2012. Kansei clustering for emotional design using a combined design structure matrix. Int. J. Ind. Ergon. 42, 416-427. doi:10.1016/j.ergon.2012.05.003

Ishihara, S., Ishihara, K., Nagamachi, M., Matsubara, Y., 1997. An analysis of Kansei structure on shoes using self-organizing neural networks. Int. J. Ind. Ergon. doi:10.1016/S0169-8141(96)00006-6

Karlsson, M., Kaulio, M., Hampf, J., Sperling, L., 1998. Eliciting Customer Requirements: Product Representations as Mediating Objects in Focus Group Interviews. Proc. from 5th Int. Prod. Dev. Conf. Como, Italy, May 26-27.

Kim, Y., Street, W.N., Russell, G.J., Menczer, F., 2005. Customer Targeting: A Neural Network Approach Guided by Genetic Algorithms. Manage. Sci. doi:10.1287/mnsc.1040.0296

Lai, H.-H., Lin, Y.-C., Yeh, C.-H., 2005. Form design of product image using grey relational analysis and neural network models. Comput. Oper. Res. 32, 2689-2711. doi:10.1016/j.cor.2004.03.021

Lai, H.-H., Lin, Y.-C., Yeh, C.-H., Wei, C.-H., 2006. User-oriented design for the optimal combination on product design. Int. J. Prod. Econ. 100, 253-267. doi:10.1016/j.ijpe.2004.11.005

Lu, W., Petiot, J.-F., 2014. Affective design of products using an audio-based protocol: Application to eyeglass frame. Int. J. Ind. Ergon. 44, 383-394. doi:10.1016/j.ergon.2014.01.004

Luce, R.D., Tukey, J.W., 1964. Simultaneous conjoint measurement: A new type of fundamental measurement. J. Math. Psychol. doi:10.1016/0022-2496(64)90015-X

Mammasse, N., Schlich, P., 2014. Adequate number of consumers in a liking test. Insights from resampling in 
seven studies. Food Qual. Prefer. 31, 124-128. doi:10.1016/j.foodqual.2012.01.009

Nagamachi, M., 2002. Kansei engineering as a powerful consumer-oriented technology for product development. Appl. Ergon. 33, 289-294. doi:10.1016/S0003-6870(02)00019-4

Nagamachi, M., Imada, A.S., 1995. Kansei Engineering: An ergonomic technology for product development. Int. J. Ind. Ergon. doi:10.1016/0169-8141(95)90025-X

Oja, E., 1992. Principal components, minor components, and linear neural networks. Neural Networks. doi:10.1016/S0893-6080(05)80089-9

Oja, E., 1982. A simplified neuron model as a principal component analyzer. J. Math. Biol. 15, 267-273. doi:10.1007/BF00275687

Paliwal, M., Kumar, U.A., 2009. Neural networks and statistical techniques: A review of applications. Expert Syst. Appl. doi:10.1016/j.eswa.2007.10.005

Park, J., Han, S.H., 2004. A fuzzy rule-based approach to modeling affective user satisfaction towards office chair design. Int. J. Ind. Ergon. doi:10.1016/j.ergon.2004.01.006

Principe, J.C., Euliano, N.R., Lefebvre, W.., 2000. Neural and Adaptative Systems: Fundamentals Throngh Simulations. Wiley and Sons, New York.

Rumelhart, D.E., Hinton, G.E., Williams, R.J., 1986. Learning internal representations by error propagation, in: Rumelhart, D.., McClelland, J.L. (Eds.), Parallel Distributed Processing: Explorations in the Microstructure of Cognition. The MIT Press, Cambridge, pp. 318-362.

Sanger, T.D., 1989. Optimal unsupervised learning in a single-layer linear feedforward neural network. Neural Networks 2, 459-473. doi:10.1016/0893-6080(89)90044-0

Sarle, W.S., 1995. Stopped Training and Other Remedies for Overfitting. Proc. 27th Symp. Interface Comput. Sci. Stat. 352-360.

Savage, S.J., Waldman, D.M., 2008. Learning and fatigue during choice experiments: a comparison of online and mail survey modes. J. Appl. Econ. 23, 351-371. doi:10.1002/jae.984

Schoormans, J.P.L., Ortt, R.J., de Bont, C.J.P.M., 1995. Enhancing concept test validity by using expert consumers. J. Prod. Innov. Manag. 12, 153-162. doi:10.1111/1540-5885.1220153

Schütte, S., 2002. Designing Feelings into Products: Integrating Kansei Engineering Methodology in Product Development, Linköpings Universitet Printings.

Shieh, M., Yan, W., Chen, C., 2008. Soliciting customer requirements for product redesign based on picture sorts and ART2 neural network. Expert Syst. Appl. 34, 194-204. doi:10.1016/j.eswa.2006.08.036

Shieh, M.D., Yan, W., Chen, C.H., 2008. Soliciting customer requirements for product redesign based on picture sorts and ART2 neural network. Expert Syst. Appl. 34, 194-204. doi:10.1016/j.eswa.2006.08.036

Shimizu, Y., Jindo, T., 1995. A fuzzy logic analysis method for evaluating human sensitivities. Int. J. Ind. Ergon. 8141.

SPSS_Inc, 2007. Clementine 12.0 Algorithms Guide.

Tsai, H.-C., Hsiao, S.-W., Hung, F.-K., 2006. An image evaluation approach for parameter-based product form and color design. Comput. Des. 38, 157-171. doi:10.1016/j.cad.2005.10.001

Venugopal, V., Baets, W., 1994. Neural Networks and Statistical Techniques in Marketing Research: AlnConceptual Comparison. Mark. Intell. Plan. 12, 30-38. doi:10.1108/02634509410065555

Vriens, M., Loosschilder, G., Rosbergen, E., Wittink, D.R., 1998. Verbal versus Realistic Pictorial 
Representations in Conjoint Analysis with Design Attributes. J. Prod. Innov. Manag. doi:10.1111/15405885.1550455

Wang, C.-H., 2014. Integrating Kansei engineering with conjoint analysis to fulfil market segmentation and product customisation for digital cameras. Int. J. Prod. Res. 53, 1-12.

doi:10.1080/00207543.2014.974840

West, P.M., Brockett, P.L., Golden, L.L., 1997. A Comparative Analysis of Neural Networks and Statistical Methods for Predicting Consumer Choice. Mark. Sci. 16, 370-391. doi:10.2307/184232

Yang, C.-C., Shieh, M.-D., 2010. A support vector regression based prediction model of affective responses for product form design. Comput. Ind. Eng. 59, 682-689. doi:10.1016/j.cie.2010.07.019 